\title{
Time Ephemeris and General Relativistic Scale Factor
}

\author{
Toshio Fukushima \\ National Astronomical Observatory of Japan, \\ 181-8588, Mitaka, Tokyo, Japan \\ email: Toshio.Fukushima@nao.ac.jp
}

\begin{abstract}
Time ephemeris is the location-independent part of the transformation formula relating two time coordinates such as TCB and TCG (Fukushima 2009). It is computed from the corresponding (space) ephemerides providing the relative motion of two spatial coordinate origins such as the motion of geocenter relative to the solar system barycenter. The time ephemerides are inevitably needed in conducting precise four dimensional coordinate transformations among various spacetime coordinate systems such as the GCRS and BCRS (Soffel et al. 2003). Also, by means of the time average operation, they are used in determining the information on scale conversion between the pair of coordinate systems, especially the difference of the general relativistic scale factor from unity such as $L_{C}$. In 1995, we presented the first numerically-integrated time ephemeris, TE245, from JPL's planetary ephemeris DE245 (Fukushima 1995). It gave an estimate of $L_{C}$ as $1.4808268457(10) \times 10^{-8}$, which was incorrect by around $2 \times 10^{-16}$. This was caused by taking the wrong sign of the post-Newtonian contribution in the final summation. Four years later, we updated TE245 to TE405 associated with DE405 (Irwin and Fukushima 1999). This time the renewed vale of $L_{C}$ is $1.48082686741(200) \times 10^{-8}$ Another four years later, by using a precise technique of time average, we improved the estimate of Newtonian part of $L_{C}$ for TE405 as 1.4808268559(6) $\times 10^{-8}$ (Harada and Fukushima 2003). This leads to the value of $L_{C}$ as $L_{C}=1.48082686732(110) \times 10^{-8}$. If we combine this with the constant defining the mean rate of TCG-TT, $L_{G}=6.969290134 \times 10^{-10}$ (IAU 2001), we estimate the numerical value of another general relativistic scale factor $L_{B}=1.55051976763(110) \times 10^{-8}$, which has the meaning of the mean rate of TCB-TT. The main reasons of the uncertainties are the truncation effect in time average and the uncertainty of asteroids' perturbation. As a compact realization of the time ephemeris, we prepared HF2002, a Fortran routine to compute approximate harmonic series of TE405 with the RMS error of 0.446 ns for the period 1600 to 2200 (Harada and Fukushima 2003). It is included in the IERS Convention 2003 (McCarthy and Petit 2003) and available from the IERS web site; http://tai.bipm.org/iers/conv2003/conv2003_c10.html.
\end{abstract}

Keywords. general relativity, ephemerides, reference systems, time

\section{References}

Fukushima, T., 1995, Astron. Astrophys., 294, 895

Fukushima, T., 2009, in Proc. IAU Symp. 261, to appear

Harada, W. \& Fukushima, T., 2003, Astron. J., 126, 2557

International Astronomical Union, 2001, in Proc. 24th General Assembly Manchester 2000, Trans. of IAU XXIVB, IAU, Paris

Irwin, A. W. \& Fukushima, T., 1999, Astron. Astrophys., 348, 642

McCarthy, D. D. \& Petit G., 2003, IERS Convention (2003), IERS Tech. Note 32, Obs. Paris

Soffel, M., Klioner, S. A., Petit, G., Wolf, P., Kopeikin, S. M., Bretagnon, P., Brumberg, V. A., Capitaine, N., Damour, T., Fukushima, T., Guinot, B., Huang, T.-Y., Lindegren, L., Ma, C., Nordtvedt, K., Ries, J. C., Seidelmann, P. K., Vokrouhlicky, D., Will, C. M., \& Xu, C., 2003, Astron. J., 126, 2687 